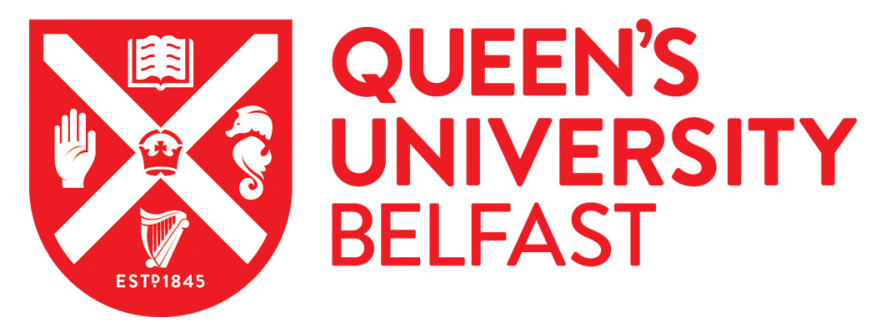

\title{
Development and validation of Fluid Structure Interaction Methods for an Oscillating Wave Surge Converter
}

Windt, C., Schmitt, P., Nicholson, J., \& Elsaesser, B. (2014). Development and validation of Fluid Structure Interaction Methods for an Oscillating Wave Surge Converter. Paper presented at International Conference on Renewable Energies Offshore, Lisbon, Portugal.

http://www.centec.tecnico.ulisboa.pt/renew2014/App_Themes/Renew2014/RENEW\%202014\%20-

\%20Final\%20Programme.pdf

Document Version:

Peer reviewed version

Queen's University Belfast - Research Portal:

Link to publication record in Queen's University Belfast Research Portal

Publisher rights

Copyright 2014 the authors.

\section{General rights}

Copyright for the publications made accessible via the Queen's University Belfast Research Portal is retained by the author(s) and / or other copyright owners and it is a condition of accessing these publications that users recognise and abide by the legal requirements associated with these rights.

Take down policy

The Research Portal is Queen's institutional repository that provides access to Queen's research output. Every effort has been made to ensure that content in the Research Portal does not infringe any person's rights, or applicable UK laws. If you discover content in the Research Portal that you believe breaches copyright or violates any law, please contact openaccess@qub.ac.uk. 


\title{
Development and Validation of Fluid Structure Interaction Methods for an Oscillating Wave Surge Converter
}

\author{
C. Windt \\ Technical University Hamburg Harburg, Hamburg, Germany
}

J. Nicholson

Aquamarine Power Ltd.

Elder House, 24 Elder Street, Edinburgh, Scotland

Dr. P. Schmitt \& Dr. B. Elsaesser

Marine Research Group, School of Planning, Architecture and Civil Engineering

Queens University, Belfast, Northern Ireland.

\begin{abstract}
The term fatigue loads on the Oyster Oscillating Wave Surge Converter is used to describe hydrostatic loads due to water surface elevation with quasi-static changes of state. Therefore a procedure to implement hydrostatic pressure distributions into finite element analysis of the structure is desired. Currently available experimental methods enable one to measure time variant water surface elevation at discrete locations either on or around the body of the scale model during tank tests. This paper discusses the development of a finite element analysis procedure to implement time variant, spatially distributed hydrostatic pressure derived from discretely measured water surface elevation. The developed method can process differently resolved (temporal and spatial) input data and approximate the elevation over the flap faces with user defined properties. The structural loads, namely the forces and moments on the body can then be investigated by post processing the numerical results. This method offers the possibility to process surface elevation or hydrostatic pressure data from computational fluid dynamics simulations and can thus be seen as a first step to a fluid-structure interaction model.
\end{abstract}

\section{INTRODUCTION}

The Oyster Oscillating Wave Surge Converter (OWSC) consists of a buoyant bottom hinged flap, which pitches back and forth in near shore ocean waves (Whittaker et al. 2007). In the statistically most common sea states, when most of the power is produced, the diffraction properties of the OWSC lead to a difference in the water surface elevation between seaward and landward flap faces (Renzi et al. 2014). This water level difference leads to a pressure gradient between the two flap faces inducing the pitching motion of the flap.

Assuming quasi-static changes of state, a direct correlation between surface elevation on the body, hydrostatic pressure and structural loads can be found (Faltinsen 1990). These loads on the structure are described by the term fatigue loads and are treated differently than loads experienced during extreme events. The quasi-static assumption is valid as long as for each time step the OWSC can be found in an equilibirum state, which can be found at low frequencies of the incoming waves. Thus non-static effects like a fast moving free surface can be neglected. Bourdier et al. 2013 showed that the quasi-static assumption can be applied with good confidence. Occurring at the statistically most common sea states the fatigue loads play a major role in terms of durability of the device and are of special interest for the design of the OWSC. The reliable definition of these load-cases and the easy and efficient application in structural simulations is required for fatigue design.

Coupling Finite Element Analysis (FEA) tools with Computational Fluid Dynamics (CFD) solvers will produce the most desirable results, enabling the numerical load definition and analysis of the structural behaviour employing Fluid-Structure Interaction simulations (FSI). However, in practice these methods are prohibitively expensive in terms of computational cost to obtain statistically relevant data (Schmitt et al. 2012). Using data obtained from experimental tank tests to create load cases for FEA simulations reduces 
the computational burden significantly.

To obtain pressure distributions during experimental tank tests pressure transducers implemented into the OWSC model could be used (Figure 3). However, in order to determine a reasonable and reliable pressure distribution across the surfaces of interest, a large number of sensors is required. Due to the submersion of the experimental model and the environmental conditions involved any instrumentation must also have a high level of ingress protection.

A simpler method to obtain structural loads experimentally using immersion probes has been presented by Bourdier et al. 2013 and will be used instead. Measuring the difference in resistance, immersion probes are able to record a time trace of the water surface elevation either on the flap surface or at an arbitrary location in the tank (Schmitt et al. 2012). Compared to pressure sensors these probes come at low cost and are simple to set-up. In this paper the probes only measure the surface elevation on the sea- and landward flap face, the highest accuracy in load derivation can thus be achieved in the two degrees of freedom (DOF) surge and pitch. These two DOF are of special interest since the OWSC is predominantly loaded in surge and pitch during normal operation. To gain load data for DOF other than these two, different experimental methods may give better results.

Implementing the experimentally gained data into an FEA simulation provides the possibility of a detailed analysis of the structural load response but requires a preprocessing environment to process experimental results. A method to fulfil this task requires access to the discretised numerical model to implement spatially and temporally distributed data.

This paper discusses the development and validation of a FEA procedure to implement spatially and temporally distributed hydrostatic pressure to a FEA model of an OWSC. Spatially distributed hydrostatic pressure is calculated for each time step of the input data and the resulting load response of the structure is post-processed. Section 2 presents the experimental tank tests performed to gain input and validation data. Section 3 presents the numerical set-up and section 4 explains the processing framework developed for the FEA simulations. Examples of a first application of the numerical method and comparison with experimental data is given in section 5. From the verification study different conclusions are drawn in section 6.

The general functionality will be proved and currently occurring limitations will be discussed. The presented results lead to future work in terms of input data quality and post-processing. In the first place the resolution of the input surface elevation data should be increased. Secondly the post-processing in th numerical model should be improved to use a more accurate structural model. If a repeated verification study then gives improved numerical results, an extraction and verification of load data not only for the

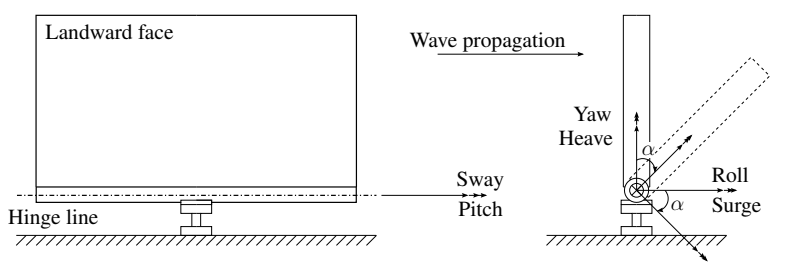

Figure 1: Declaration of Degrees of Freedom

surge and pitch DOF as well as a application for a moving OWSC model is desired.

\section{EXPERIMENTAL TANK TESTS}

Within this paper the six different DOF, Surge, Sway, Heave, Roll, Pitch and Yaw are defined as shown in Figure 1.

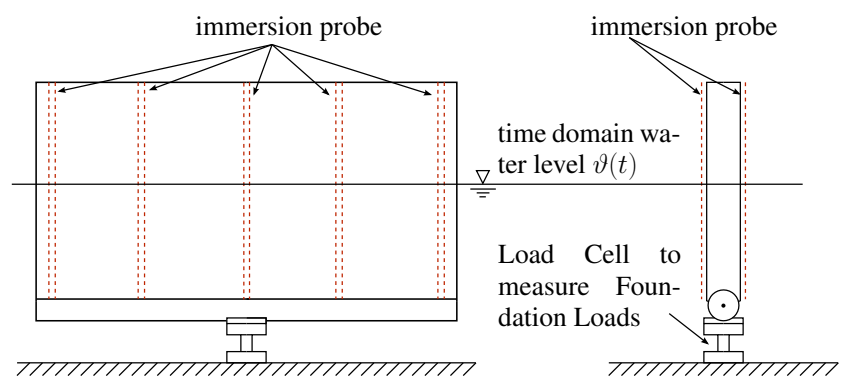

Figure 2: Schematic Drawing of Test Setup to gain Verification Data

To gain input and verification data for the initial tests of the developed procedure, experimental tank tests with a $40^{t h}$ scale model of the OWSC were performed at the wave tank in Queen's University Belfast. The model was fixed around the hinge to simplify these initial tests. A schematic drawing of the experimental set-up is shown in Figure 2. The OWSC tank model was exposed to water waves and surface elevation and foundation loads were measured. The water surface elevation directly on the flap surface was measured with immersion probes. To keep the data processing simple, only two immersion probes were installed on each flap side. Additionally to the surface elevation, the loads on the structure were measured with the help of a six degree of freedom load cell located at the base of the flap (Figure 3). This load data is used to verify the developed FEA procedure by comparing foundation load data with numerically calculated loads (section 5). Time traces of surface elevation and loads are presented in section 5 .

\section{NUMERICAL SETUP}

To perform the finite element analysis simulations the OpenSource FEA tool Code Aster was used. This framework provides thermo-mechanical (non-)linear solvers and utilities which can be accessed with the help of pre-defined commands or via Fortran and Python interfaces.

To get reliable data from the performed simulations, a numerical OWSC model is needed which 


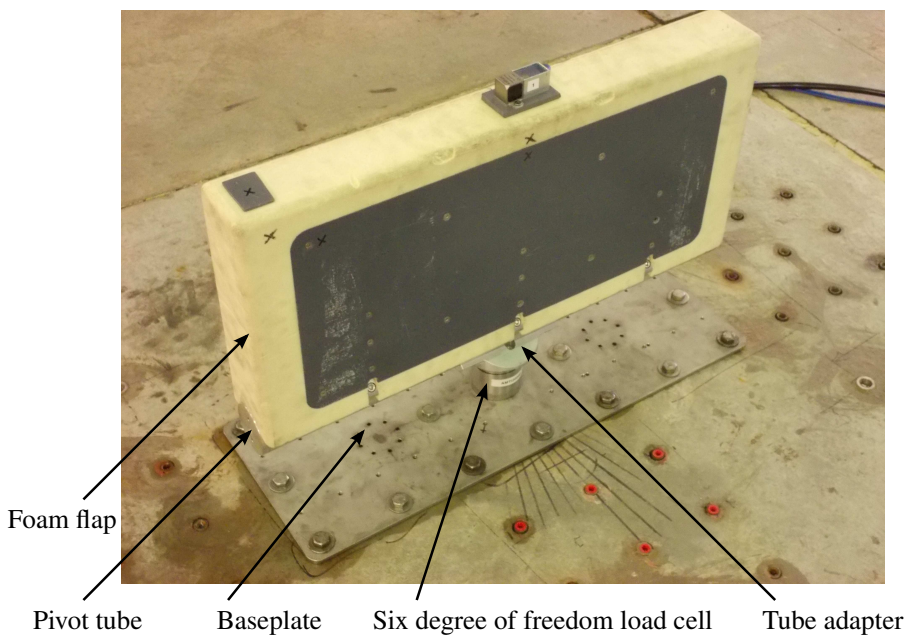

Figure 3: Physical Tank Model of an OWSC

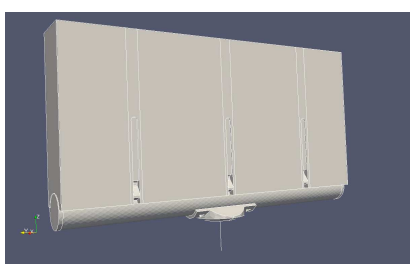

Figure 4: Simplified Numerical Model of an OWSC

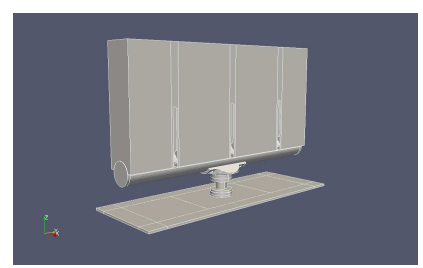
Model of an OWSC
Figure 5: Full Numerical

mimics the physical one (Figure 3 ). To prove the reliabilty of the numerical model a comparison study between numerical and physical natural frequencies was performed for the dry structure (Table 1) (Schwarz and Richardson 1999). Impact hammer tests were carried out to obtain the natural frequencies of the tank model (Kistler 2014). To gain numerical natural frequencies, a modal analysis with the help of Code_Aster has been performed.

Very good agreement between physical and numerical natural frequencies can be achieved when comparing the results of a detailed numerical OWSC model (Figure 5).However due to current limitations such a detailed model can not be used. The comparison between experimental and numerical load data requires the extraction of numerically gained loads by post-processing the performed simulations. During the numerical calculations loads are calculated for the nodes of the discretized model. Hence to extract loads on a certain area of this model, an integration is needed. To gain numerical loads in areas equivalent to load areas of the physical model, such an integration is necessary. Preliminary tests revealed limitations in terms of load post processing. The integration of loads over nodes did not yield reliable results. Additionally these tests showed, that loads can only be extracted accurately at single boundary nodes of the investigated model.The numerical load-cell model had thus to be simplified to a beam element (with coinciding geometrical and mechanical properties) and neglecting the baseplate as well as the foundation (Figure 4). This simplified model does not exactly mimic the physical model. Table 1 shows the deviations in natural frequency of the two numerical models from ex-
Table 1: Relative Deviation between Natural Frequency of Physical and Numerical Flap Models

\begin{tabular}{lll}
\hline & Complete Model & Simplified Model \\
\hline Roll & $3 \%$ & $20 \%$ \\
Pitch & $0 \%$ & $7 \%$ \\
Yaw & $5 \%$ & $0 \%$ \\
\hline
\end{tabular}

perimental data. The maximum difference observed between experimental and numerical eigenfrequency increases from $5 \%$ in yaw for the detailed model to $20 \%$ in the roll mode for the simplified model. Since the main loading investigated in this paper is normal to the flap faces and will thus impose pitching moments, the simplified model is still deemed acceptable for initial tests.

\section{FEA PROCEDURE}

During the FEA preparation general procedures for the performance of a static simulation are carried out. The meshes of the different model parts are read in, an overall structural model is defined and boundary conditions as well as material properties are set. For all these procedures the use of the provided CodeAster functions is straightforward.

For the computation of the pressure distribution, python functions have been developed and implemented into the FEA simulations. In a first step, experimental data of measured surface elevation is passed to the simulation in a compatible format. Since the data is measured at spatially discrete locations a curve fit through the discrete elevation values, with a user defined function, is performed for all time steps. Thus surface elevation values are defined over the complete flap width. For the special case of just one immersion probe (on each flap side) a constant water level is assumed over the flap width. If data of two probes on each flap side are available the surface elevation will be averaged and again a spatially constant water level is assumed. With the help of the fitted immersion data the pressure for each surface panel is derived (Figure 6).

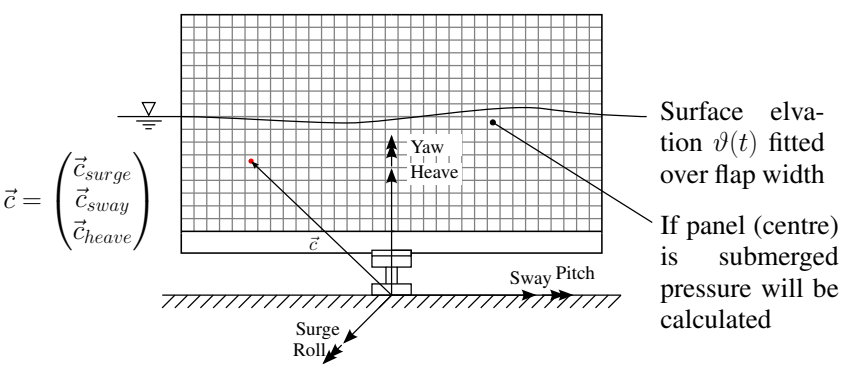

Figure 6: Schematic Drawing of Pressure Determination for Mesh Panels

With information about panel centre location (panel centre coordinates $\vec{c}_{\text {surge }}, \vec{c}_{\text {sway }}, \vec{c}_{\text {heave }}$ ) and water surface elevation along the flap face, it can be determined if a certain panel is submerged by simply comparing water level and panel centre coordinate. If 
the panel is submerged $\left(\vartheta>\vec{c}_{\text {heave }}\right.$ ) the pressure is calculated by using equation 1 .

$p_{i, \text { panel }}=\rho \cdot g \cdot\left(\vartheta(t)_{i}-\vec{c}_{\text {heave }}\right)$

The hydrostatic pressure for each panel $p_{i, p a n e l}$ at time step $i$ is defined as a function of the fluid density $\rho$, the gravitational acceleration $g$, the surface elevation $\vartheta(t)_{i}$ at time step $i$ and the panel location in heave $c_{\text {heave. }}$. With the defined pressure on submerged surface panels of the structure, the quasi-static load response of the structure can be calculated.

Together with the model and boundary conditions defined in the phase of the FEA preparation, information about the hydrostatic pressure are fed into a static solver and the calculations of the structural load response is performed. This procedure (deriving pressures for surface panels and calculating the static solution) is performed for each time step of the input time trace. The overall procedure for the implementation of hydrostatic pressure due to surface elevation is illustrated in Figure 7.

\section{NUMERCIAL RESULTS AND DISCUSSION}

To test the reliability of the developed implementation, numerical and experimental loads are compared. The static load response of the flap structure to the hydrostatic pressure distribution has been calculated for 41 time steps over 2 seconds. The time trace of the input surface elevation can be seen in Figure 14. Surface elevation from the mean water level of the two immersion probes is plotted over time for each side of the flap. Surface elevation on the seaward flap face shows about two times larger elevation amplitudes than on the landward side. The wave crest on the seaward side precedes the landward side by about $0.3 \mathrm{~s}$. Significant differences of up to $70 \mathrm{~mm}$ can be observed between data from immersion probes at the landward and seaward side at $t=0.1 \mathrm{~s}$ (cf. fig. 14).

Applying the developed procedure results in the averaged constant water level as shown in Figures 17 to 19, the derived spatial pressure distribution (Figure 8 to 13 ) and the the resulting structural load response (Figures 15 and 16).

Comparing the surface elevation time trace and pressure distribution, reasonable agreement can be found. At the time $t=0 \mathrm{~s}$ a constant water level of $0.343 \mathrm{~m}$ at the seaward flap face can be calculated from the measured data. Applying equation 1 this leads to a hydrostatic pressure of $2174 P a$ at the lower edge of the flap face. This pressure has been calculated by the developed tool and can be found in Figure 9. Looking at the surface elevation at $t=0.5 \mathrm{~s}$ higher elevation can be seen on the landward flap face. Hence higher pressures are calculated on this flap face (Figure 10 and 11). At $t=1.6 \mathrm{~s}$ the measured surface elevation on both sides of the flap show the same values, hence an identical pressure distribution can be seen in Figures 12 and 13 for landward and seaward faces.
Since the (fatigue) loads acting on the structure arise from pressure differences between seaward and landward flap faces, foundation loads of around $0 N$ and $0 N m$ are expected at $t=1.6 s$. This can be seen in the experimental as well as the numerical data in Figures 15 and 16. The time traces of force in surge (Figure 15) and torque in pitch (Figure 16) generally show a good match between experimental and numerical results in phase, but significant differences in amplitude can be observed. At $t=0.05 \mathrm{~s}$ the numerical results for torque show a value of $18.2 \mathrm{Nm}$. Compared to the experimentally determined value of $9.1 \mathrm{Nm}$ resulting in a deviation of $50 \%$. At the lowest value of the numerical torque $(t=0.54 \mathrm{~s})$ a value of $-10.6 \mathrm{Nm}$ has to be compared to a physical value of $-6.4 \mathrm{Nm}$ leading to a deviation of $40 \%$. Such an overshooting of the numerically calculated load response can also be seen in the force time trace in the surge DOF but with lower relative deviations. At $t=0.05 \mathrm{~s}$ a force of $85.2 \mathrm{~N}$ has been calculated whereas in the experiments a force of $60.3 \mathrm{~N}$ was measured, leading to a deviation of $30 \%$. At $t=0.54 \mathrm{~s},-53.7 \mathrm{~N}$ has been calculated and $-48.3 \mathrm{~N}$ measured, hence a deviation of $10 \%$ can be found at this time step. The listed results can be seen in table 2 .

Looking at the time traces of surface elevation (Figure 14), force (Figure 15) and torque (Figure 16), highest deviations between measured and calculated load response can be found at time steps, where different values of surface elevation are recorded on the same flap side. This mainly occures at surface elevation peaks $(t=0.1 s, t=0.15 s, t=1.5 s)$. At time steps before and after such elevation peaks reasonable results for the calculated loads can be found. Since the phase of the calucalted force and torque also shows good agreement the main source of deviation is related to the inaccuracy of the measured surface elevation. As mentioned before, for these initial tests surface elevation has only been measured at two discrete locations on each flap side and averaged values have been applied over the width of the flap. This set-up can neither take into account any curvature of the water surface over the width of the flap, nor is it possible to filter out small disturbances of the water surface. Since the loading is the differential of the two, any error in the surface elevation will have a major impact on the resulting force. Bourdier et al. 2013 used three wave probes on one half of each flap face and obtained good results, proving the validity of the concept for small waves of $1-2 m$ elevation. The functionality to use three or more wave probes and the interpolation of a non-linear surface elevation over the flap width has already been implemented and will be tested in the near future. For larger waves Bourdier et al. 2013 observed an overestimation of the peak pressure. For the presented validation study such large waves have been investigated, so that an overshooting can also originate in wave amplitude.

Due to the applied simplifications in the numeri- 


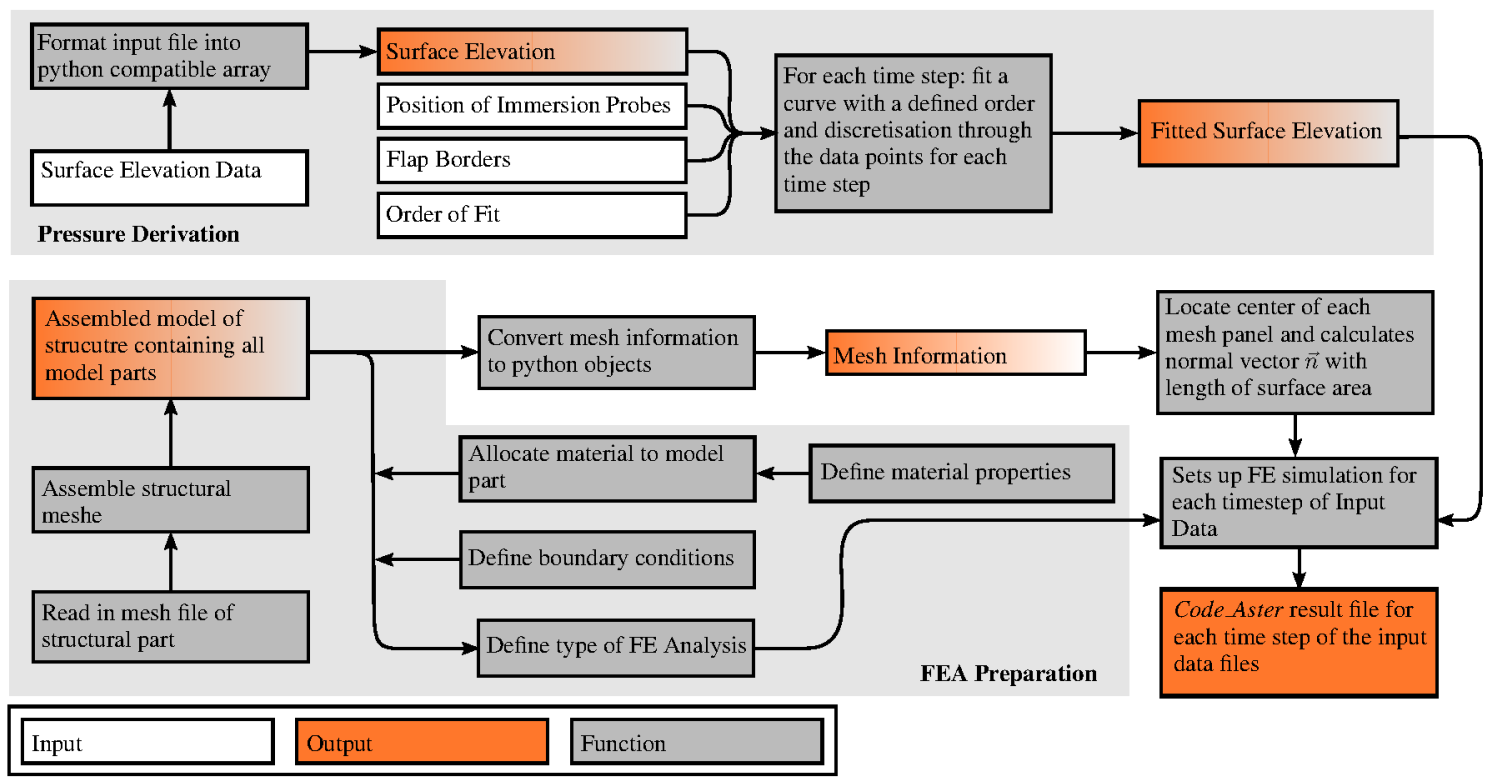

Figure 7: Flowchart representation of data processing steps

Table 2: Numerical and Experimental Loads at Different Timesteps

\begin{tabular}{|c|c|c|c|c|}
\hline & \multicolumn{2}{|l|}{ Time $t=0.05 \mathrm{~s}$} & \multicolumn{2}{|l|}{ Time $t=0.54 \mathrm{~s}$} \\
\hline & Force in Surge & Torque in Pitch & Force in Surge & Torque in Pitch \\
\hline Numerical Results & $85.2 N$ & $18.2 \mathrm{Nm}$ & $-53.7 N$ & $-10.6 \mathrm{Nm}$ \\
\hline Experimental Results & $60.3 N$ & $9.1 N m$ & $-48.3 N$ & $-6.4 N m$ \\
\hline Relative Deviation & $30 \%$ & $50 \%$ & $10 \%$ & $40 \%$ \\
\hline
\end{tabular}

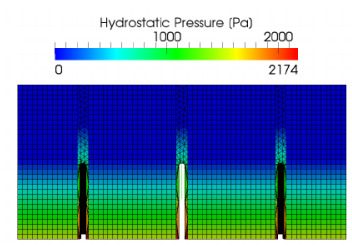

Figure 8: Pressure on Landward Flap Face at $t=0 \mathrm{~s}$

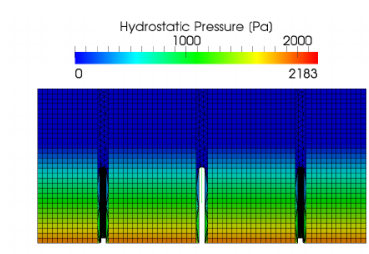

Figure 10: Pressure on Landward Flap Face at $t=0.5 \mathrm{~s}$

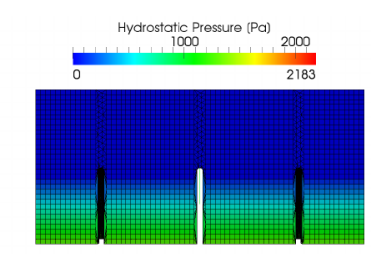

Figure 12: Pressure on Landward Flap Face at $t=1.6 \mathrm{~s}$

cal model, differences in the location of data acquisition can be seen as a further origin of load data deviation. Because of the aforementioned current nu- merical limitations (section 3) the loads have to be extracted at the boundary node at the bottom of the load cell. At this point the numerical geometry differs from the geometry where the strain gauges are attached in the physical model. Additionally the lever arm of the implemented hydrostatic pressure changes with the restricted numerical data acquisition location and higher deviations between measured and calculated torque (in pitch) compared to deviations between measured and calculated force (in surge) might thus be explained.

Although not included in this paper, the resulting force from the assumed pressure distribution is evaluated independently during preprocessing and compared to the resulting load-cell data to ensure sufficient mesh resolution.

Some deviations between the structural dynamic behaviour of the numerical and the physical model had to be expected, since hammer tests revealed some discrepancies. Although only quasi-static changes of state are considered and a static solver is applied to calculate the load response of the structure, the differences in the natural frequency reduce the confidence in obtained data and suggest to improve the representation of the physical model. 
- A procedure to apply time variant hydrostatic pressure based on experimental water surface elevations on the assumption of quasi-static changes of state has been developed and shows generally good agreement for a simplified test case

- Observed deviations are mainly believed to stem from insufficient accuracy and resolution of the experimental surface elevation

- Simplifications of the numerical model also lead to considerable differences when comparing eigen-frequencies of experimental and numerical models

\section{REFERENCES}

Bourdier, S., K. Abdulla, A. Henry, \& T. Whittaker (2013). Derivation of wave loads for the design of oscillating wave surge converters. In European Wave and Tidal Energy Conference. European Wave and Tidal Energy Conference.

Faltinsen, O. (1990). Sea Loads on Ship and Offshore Structures. Cambridge University Press.

Kistler (2014). Theory of the hammer method in modal testing. Kistler News 2.

Renzi, E., K. Doherty, A. Henry, \& F. Dias (2014). How does oyster work? the simple interpretation of oyster mathematics. Journal of Mechanics.

Schmitt, P., S. Bourdier, D. Sarkar, E. Renzi, F. Dias, K. Doherty, T. Whittaker, \& J. van 't Hoff (2012). Hydrodynamic loaload on a bottom hinged oscillating wave surge converter. In International Offshore and Polar Engineering Conference.

Schmitt, P., K. Doherty, D. Clabby, \& T. Whittaker (2012). The opportunities and limitations of using cfd in the development of wave energy converters. In Marine \& Offshore Renewable Energy.

Schwarz, B. \& M. Richardson (1999). Experimental modal analysis. CSI Reliability Week.

Whittaker, T., D. Collier, \& M. Folley (2007). The development of oyster - a shallow water surging wave energy converter. In 7th Annual European Wave \& Tidal Energy Conference.

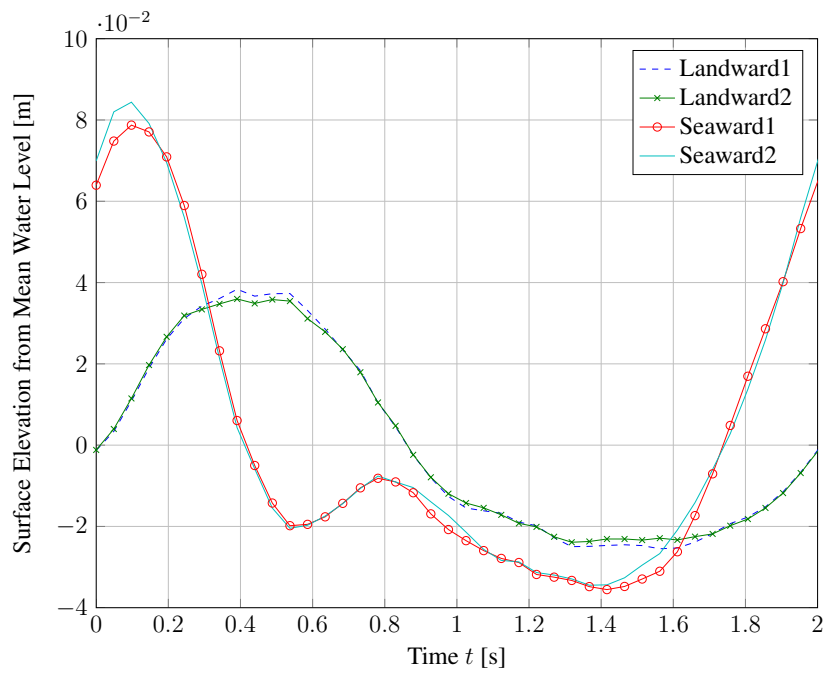

Figure 14: Time Trace of Landward and Seaward Surface Elevation

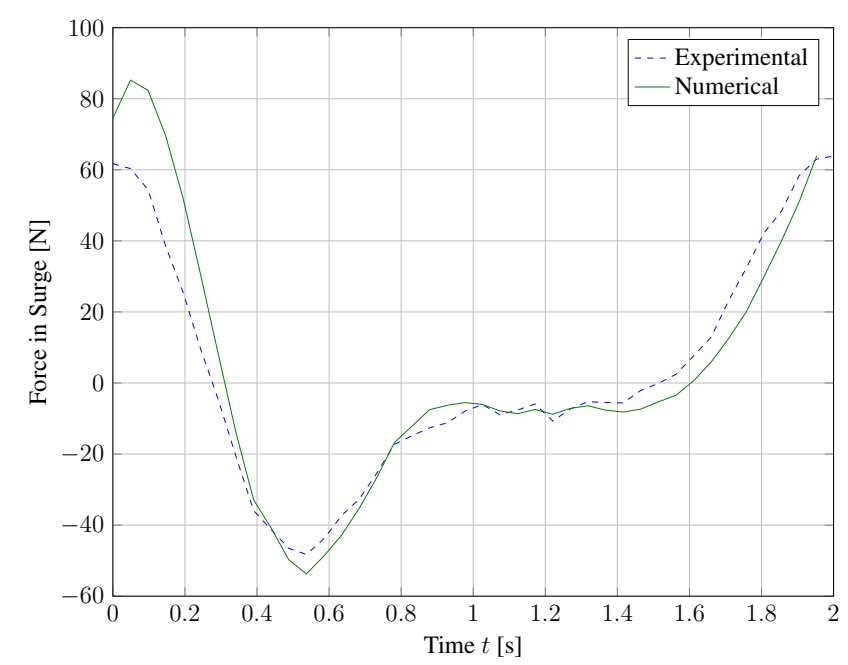

Figure 15: Comparison between measured and calculated Force in Surge

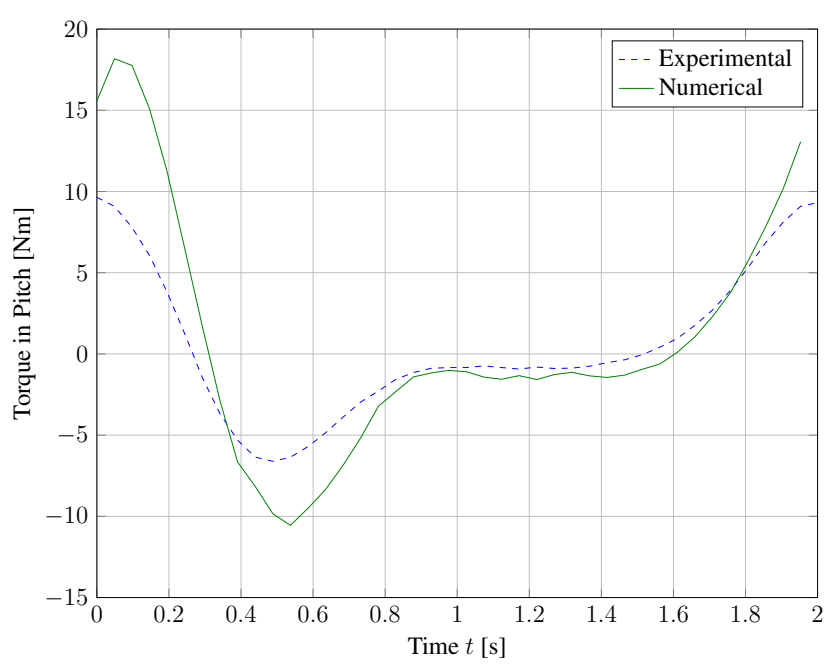

Figure 16: Comparison between measured and calculated Torque in Pitch 


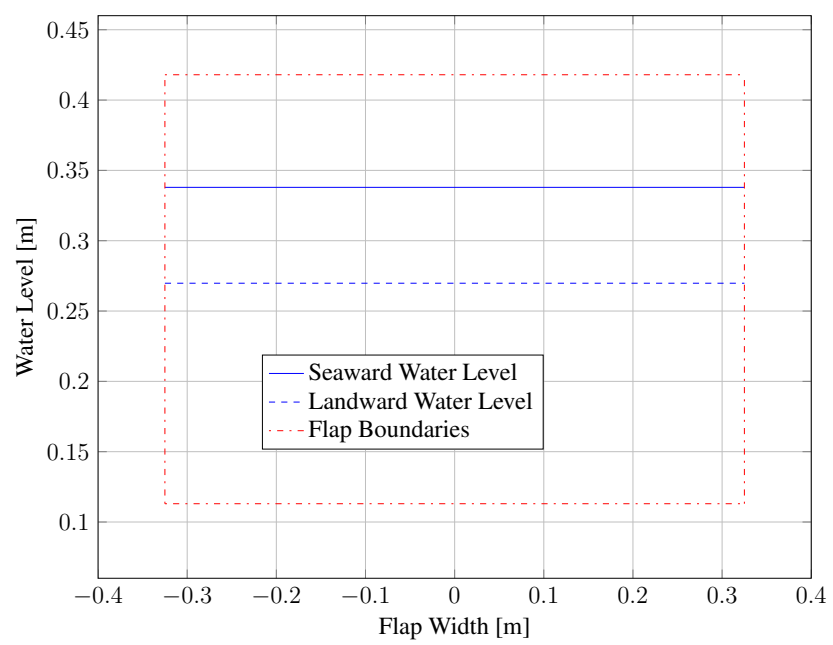

Figure 17: Fitted Surface Elevation on Sea- and Landward Flap Face at $t=0 \mathrm{~s}$

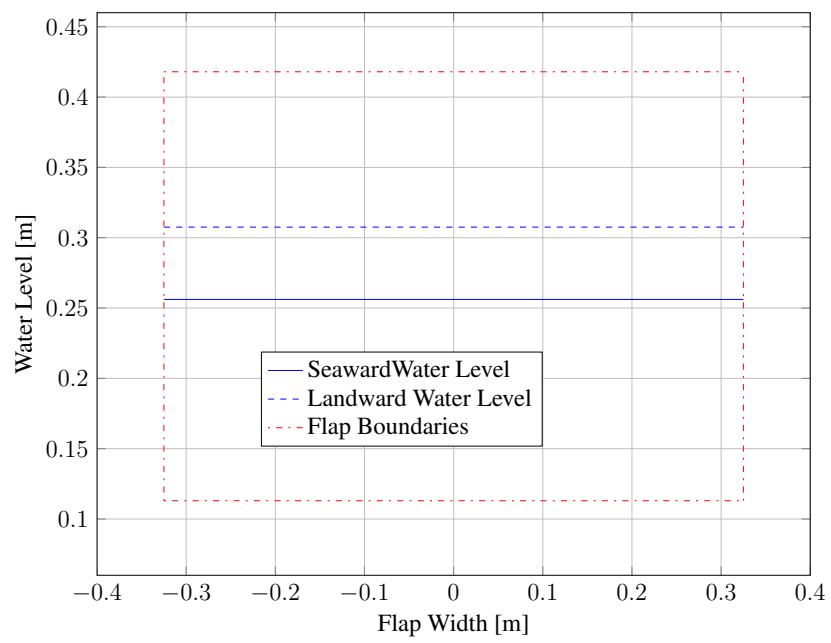

Figure 18: Fitted Surface Elevation on Sea- and Landward Flap Face at $t=0.5 \mathrm{~s}$

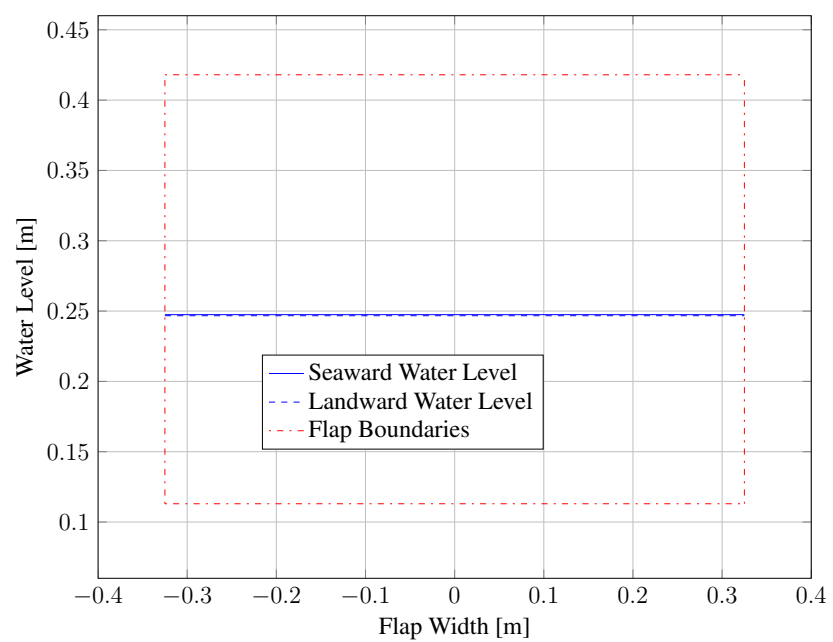

Figure 19: Fitted Surface Elevation on Sea- and Landward Flap Face at $t=1.6 \mathrm{~s}$ 